\title{
ON THE ARG MIN MULTIFUNCTION FOR LOWER SEMICONTINUOUS FUNCTIONS
}

\author{
GERALD BEER AND PETAR KENDEROV
}

(Communicated by Doug W. Curtis)

\begin{abstract}
The epi-topology on the lower semicontinuous functions $L(X)$ on a Hausdorff space $X$ is the restriction of the Fell topology on the closed subsets of $X \times R$ to $L(X)$, identifying lower semicontinuous functions with their epigraphs. For each $f \in L(X)$, let $\arg \min f$ be the set of minimizers of $f$. With respect to the epi-topology, the graph of arg min is a closed subset of $L(X) \times X$ if and only if $X$ is locally compact. Moreover, if $X$ is locally compact, then the epi-topology is the weakest topology on $L(X)$ for which the arg min multifunction has closed graph, and the operators $f \rightarrow f \vee g$ and $f \rightarrow f \wedge g$ are continuous for each continuous real function $g$ on $X$.
\end{abstract}

1. Introduction. An extended real valued function on a topological space $X$ is called lower semicontinuous (l.s.c.) if for each real $\alpha,\{x: f(x)>\alpha\}$ is an open subset of $X$. Equivalently [11], $f$ is lower semicontinuous if its epigraph epi $f=\{(x, \alpha): \alpha \in R$ and $\alpha \geq f(x)\}$ is a closed subset of $X \times R$. In the sequel, we denote the lower semicontinuous functions on $X$ by $L(X)$, and the real valued continuous functions on $X$ by $C(X)$. The fundamental notion of convergence for lower semicontinuous functions proceeds from the identification of such a function with its epigraph [11].

DEFINITION. A net $\left\langle f_{\lambda}\right\rangle$ of lower semicontinuous functions on $X$ is said to be epiconvergent to a lower semicontinuous function $f$ if epi $f=\operatorname{Lsepi} f=\operatorname{Li}$ epi $f$, where Ls epi $f$ (resp. Li epi $f$ ) consists of all points $(x, \alpha)$ in $X \times R$ such that each neighborhood of $(x, \alpha)$ meets epi $f_{\lambda}$ frequently (resp. eventually).

Epiconvergence may be characterized locally as follows: at each $x$ in $X$, both of the following conditions must hold:

(i) whenever $\alpha<f(x)$, there exists a neighborhood $U$ of $x$ such that $f_{\lambda}^{-1}((\alpha, \infty])$ contains $U$ eventually;

(ii) whenever $\alpha>f(x)$ and $U$ is a neighborhood of $x$, then $f_{\lambda}^{-1}([-\infty, \alpha))$ meets $U$ eventually.

When $X$ is first countable and $\left\langle f_{\lambda}\right\rangle$ is a sequence of l.s.c. functions, then by virtue of Proposition 1.14 and Theorem 1.39 of [1], epiconvergence may be characterized by the conjunction of these very tangible local conditions: at each $x$ in $X$,

(i) whenever $\left\langle x_{\lambda}\right\rangle \rightarrow x$, then $\underline{\lim } f_{\lambda}\left(x_{\lambda}\right) \geq f(x)$;

(ii) there exists a sequence $\left\langle x_{\lambda}\right\rangle$ convergent to $x$ for which $\lim f_{\lambda}\left(x_{\lambda}\right)=f(x)$.

Received by the editors September 16, 1986.

1980 Mathematics Subject Classification (1985 Revision). Primary 26A15, 49A27; Secondary $54 \mathrm{C} 60$.

Key words and phrases. arg min multifunction, lower semicontinuous function, epiconvergence, epi-topology, Fell topology, function lattice, locally compact Hausdorff space. 
It is a straightforward exercise to modify the standard example [12] of a sequence of step functions convergent nowhere pointwise on $[0,1]$, yet convergent in measure to the zero function, to obtain an epiconvergent sequence in $C([0,1])$ convergent nowhere pointwise.

Epiconvergence for convex functions in finite dimensions was first considered by Wijsman [17], who proved that if $\left\langle f_{n}\right\rangle$ is a sequence of l.s.c. convex functions epiconvergent to $f$, then epiconvergence of their Young-Fenchel transforms to that of $f$ also occurs. Subsequently, epiconvergence has been applied to nonlinear optimization problems in a variety of settings $[1,2,4,13,16,18]$.

It is well known ([5], or more generally, [7] or $\S 4.5$ of $[\mathbf{1 0}])$ that epiconvergence is topological in the sense of Kelley [9], provided $X$ is a locally compact Hausdorff space. The topology of epiconvergence, which we call the epi-topology $\tau_{e}$, turns out to be the restriction of the Fell topology [8] on the closed subsets of $X \times R$ to the lower semicontinuous functions, as identified with their epigraphs. A subbase for the epi-topology on $L(X)$ consists of all sets of the form

$$
W^{-}=\{f \in L(X): \text { epi } f \cap W \neq \varnothing\}
$$

and

$$
\left(K^{c}\right)^{+}=\left\{f \in L(X): \text { epi } f \subset K^{c}\right\}
$$

where $W$ runs over the open subsets of $X \times R$, and $K$ runs over the compact subsets of $X \times R$. Now if $K_{1}$ and $K_{2}$ are compact subsets of $X \times R$, we have $\left(\left[K_{1} \cup K_{2}\right]^{c}\right)^{+}=\left(K_{1}^{c}\right)^{+} \cap\left(K_{2}^{c}\right)^{+}$. As a result, a base for the topology $\tau_{e}$ consists of all sets of the form $\left(\bigcap_{i=1}^{n} W_{i}^{-}\right) \cap\left(K^{c}\right)^{+}$where $\left\{W_{1}, \ldots, W_{n}\right\}$ is a family of open subsets of $X \times R$, and $K$ is a compact subset of $X \times R$ disjoint from $\bigcup W_{i}$. If $X$ is locally compact, $\left\langle L(X), \tau_{e}\right\rangle$ is a compact Hausdorff space, and if $X$ is also second countable, then $\left\langle L(X), \tau_{e}\right\rangle$ is metrizable [1]. It should be noted that if $X$ is first countable but not necessarily locally compact, then epiconvergence of sequences is still convergence with respect to this topology.

If $f \in L(X)$ we denote by $\arg \min f$ the (possibly empty) closed subset of $X$ consisting of the minimizers of $f$; that is,

$$
\arg \min f=\{x \in X: f(x)=\inf f(X)\} .
$$

$\arg$ min may be considered as a set valued function, or multifunction, from $L(X)$ to $X[\mathbf{1 1}]$; from this point of view, its graph [10] as a subset of $L(X) \times X$ consists of the set of all $(f, x)$ such that $x$ is a minimizer of $f$. Now any reasonable topology $\tau$ on $L(X)$ should have the property that the graph of $\arg \min$ is closed, i.e., if $\left\langle f_{\lambda}\right\rangle$ is $\tau$-convergent to $f$ and $x_{\lambda} \in \arg \min f_{\lambda}$ for each $\lambda$, then the convergence of $\left\langle x_{\lambda}\right\rangle$ to $x$ implies $x \in \arg \min f$. It is well known (see, e.g., formula 3.38 of [11]) that this is the case if $X=R^{n}$ when $\tau$ is the epi-topology. We show here that closedness of the graph of arg min with respect to the epi-topology actually characterizes local compactness for the domain space $X$. Moreover, we show in this context that the epi-topology is, essentially, the weakest topology on $L(X)$ such that the graph of arg min is closed.

\section{Results.}

THEOREM 1. Let $X$ be a Hausdorff space. The following are equivalent:

(1) $X$ is locally compact;

(2) $\arg \min :\left\langle L(X), \tau_{e}\right\rangle \rightarrow X$ has a closed graph. 
Proof. $(1) \rightarrow(2)$. We show that if $\left(f, x_{0}\right)$ does not lie in the graph of arg min, there exists a neighborhood of $\left(f, x_{0}\right)$ in $L(X) \times X$ which fails to meet the graph. Since $x_{0}$ is not a minimizer of $f$, there exists $x_{1} \in X$ with $f\left(x_{1}\right)<f\left(x_{0}\right)$. Let $\alpha$ lie strictly between $f\left(x_{1}\right)$ and $f\left(x_{0}\right)$; since $\{x: f(x)>\alpha\}$ is open and $X$ is a locally compact Hausdorff space, there exists a compact neighborhood $C$ of $x_{0}$ not containing $x_{1}$ such that $C \subset\{x: f(x)>\alpha\}$. Set $K=C \times\{\alpha\}$ and $W=$ $C^{c} \times(-\infty, \alpha)$. Clearly, $\left(f, x_{0}\right) \in\left(W^{-} \cap\left(K^{c}\right)^{+}\right) \times C$. We claim that if $(g, x) \in$ $\left(W^{-} \cap\left(K^{c}\right)^{+}\right) \times C$, then $(g, x)$ fails to lie in the graph of arg min. First, $x \in C$ and $g \in\left(K^{c}\right)^{+}$jointly imply that $g(x)>\alpha$; on the other hand, $g \in W^{-}$says that for some $z \in C^{c}$, we have $g(z)<\alpha$. Thus, $x$ is not a minimizer of $g$.

$(2) \rightarrow(1)$. Suppose $(1)$ fails, i.e., at some point $x_{0}$ of $X$, the topology fails to have a local base of compact neighborhoods. It is easy to verify [6] that $x_{0}$ can have no compact neighborhood whatsoever. Choose $p \neq x_{0}$, and let $f$ be the indicator function of $\{p\}$; that is

$$
f(x)= \begin{cases}0 & \text { if } x=p \\ \infty & \text { if } x \neq p\end{cases}
$$

Clearly, $x_{0} \notin \arg \min f$. We claim that each neighborhood of $\left(f, x_{0}\right)$ meets the graph of arg min. To this end, let $\left(\bigcap_{i=1}^{n} W_{i}^{-}\right) \cap\left(K^{c}\right)^{+}$be a neighborhood of $f$ and let $U$ be a neighborhood of $x_{0}$. Now $\pi_{X}(K)$ is compact, and since $U$ is noncompact, $U \backslash\left(\pi_{X}(K) \cup\{p\}\right)$ must be an infinite set. Select $u_{0}$ in this difference, and define $g \in L(X)$ by

$$
g(x)= \begin{cases}-1 & \text { if } x=u_{0} \\ 0 & \text { if } x=p \\ \infty & \text { otherwise }\end{cases}
$$

Note that

$$
\begin{aligned}
\text { epi } g & =\left(\left\{u_{0}\right\} \times[-1, \infty)\right) \cup(\{p\} \times[0, \infty)] \\
& \supset\{p\} \times[0, \infty)=\mathrm{epi} f
\end{aligned}
$$

and since epi $f$ meets $W_{i}$ for each $i \in\{1, \ldots, n\}$, so must epi $g$. Also, by the choice of $u_{0}$, epi $g \subset K^{c}$. It is obvious that $u_{0} \in \arg \min g$. Thus,

$$
\left[\left(\bigcap_{i=1}^{n} W_{i}\right) \cap\left(K^{c}\right)^{+}\right] \times U
$$

contains a point of the graph of arg min, namely, $\left(g, u_{0}\right)$. As a result, (2) fails.

We intend to show that when $X$ is a locally compact Hausdorff space, $\tau_{e}$ is the smallest topology on $L(X)$ such that arg min has a closed graph, and the lattice operations on $L(X)$ are moderately well behaved. Of course, $L(X)$ forms a lattice with respect to ordinary function supremum and infimum, but it does not form a topological lattice. In particular, $\vee$ need not be continuous on $C(X) \times C(X)$, nor need it be continuous in each variable separately on $L(X) \times L(X)$. Continuity of the lattice operations has been considered by Dal Maso [3]; we include a self-contained treatment.

EXAMPLE 1. Let $X=[0,1]$, and for each $n \in Z^{+}$, let $f_{n}: X \rightarrow R$ be the continuous function whose graph is the polygonal path connecting these points in 
succession:

$$
(0,0),\left(\frac{1}{2 n}, 1\right),\left(\frac{2}{2 n}, 1\right),\left(\frac{3}{2 n}, 1\right), \ldots,\left(\frac{2 n-1}{2 n}, 1\right),(1,0) .
$$

Let $g_{n}=1-f_{n}$; evidently, both $\left\langle f_{n}\right\rangle$ and $\left\langle g_{n}\right\rangle$ epiconverge to the zero function. Now for each $x,\left(f_{n} \vee g_{n}\right)(x) \geq 1 / 2$, and $\left\{x:\left(f_{n} \vee g_{n}\right)(x)=1 / 2\right\}$ is $1 / 2 n$-dense in $[0,1]$. As a result, $\left\langle f_{n} \vee g_{n}\right\rangle$ epiconverges to the function assigning the value $1 / 2$ to each $x$, and not to $\left(\tau_{e}-\lim f_{n}\right) \vee\left(\tau_{e}-\lim g_{n}\right)$.

EXAMPLE 2. Let $X=[0,1]$, let $f$ be the indicator function of $\{0\}$, and let $f_{n}$ be the indicator function of $\{1 / n\}$. Then for each $n, f \vee f_{n}$ assigns infinity to each point of $[0,1]$. Since $\left\langle f_{n}\right\rangle$ epiconverges to $f$, we have $f=f \vee\left(\tau_{e}-\lim f_{n}\right) \neq \tau_{e}-\lim \left(f \vee f_{n}\right)$.

LEMMA A. Let $X$ be a Hasusdorff space and let $L(X)$ be the lower semicontinuous functions on $X$, equipped with $\tau_{e}$. Then $\wedge: L(X) \times L(X) \rightarrow L(X)$ is continuous.

PROOF. We show that the inverse image of each subbasic open set is open. First, let $K$ be a compact subset of $X \times R$, and suppose that $f \wedge g \in\left(K^{c}\right)^{+}$. This means epi $f \cup$ epi $g \subset K^{c}$, whence both $f \in\left(K^{c}\right)^{+}$and $g \in\left(K^{c}\right)^{+}$. In $L(X) \times L(X),\left(K^{c}\right)^{+} \times\left(K^{c}\right)^{+}$is a neighborhood of $(f, g)$, and it is obvious that $\left(K^{c}\right)^{+} \wedge\left(K^{c}\right)^{+} \subset\left(K^{c}\right)^{+}$.

Now suppose that $W$ is an open in $X \times R$ and $f \wedge g \in W^{-}$. This means either epi $f \cap W \neq \varnothing$ or epi $g \cap W \neq \varnothing$. By symmetry, it suffices to look at the first case. If epi $g \neq \varnothing$, then $f \wedge g \in W^{-} \wedge(X \times R)^{-} \subset W^{-}$, whereas if epi $g=\varnothing$ and $x_{0} \in X$, then $f \wedge g \in W^{-} \wedge\left(\left\{\left(x_{0}, 0\right)\right\}^{c}\right)^{+} \subset W^{-}$.

LEMMA B. Let $X$ be a Hausdorff space and let $L(X)$ be the lower semicontinuous functions on $X$, equipped with $\tau_{e}$. Then for each $g \in C(X), \phi_{g}: L(X) \rightarrow L(X)$ defined by $\phi_{g}(f)=f \vee g$ is continuous.

ProOF. Let $K$ be a compact subset of $X \times R$, and suppose that $f$ is a lower semicontinuous function satisfying $\phi_{g}(f) \in\left(K^{c}\right)^{+}$. Let $C=\pi_{X}(K)$ and define $h: C \rightarrow R$ by $h(x)=\sup \{\alpha:(x, \alpha) \in K\}$. Note that a lower semicontinuous function lies in $\left(K^{c}\right)^{+}$if and only if it exceeds $h$ on $C$. It is easy to check that $-h$ is l.s.c., and since $L(C)$ forms a cone, $g-h \in L(C)$. As a result,

$$
F=\{x \in C: g(x)-h(x) \leq 0\}
$$

is compact, so that $K_{1}=K \cap(F \times R)$ is compact. We claim that $\left(K_{1}^{c}\right)^{+}$is a neighborhood of $f$ mapped by $\phi_{g}$ into $\left(K^{c}\right)^{+}$. To see that $f \in\left(K_{1}^{c}\right)^{+}$we verify that $f$ exceeds $h$ on $F$ : if $x \in F$, then $g(x) \leq h(x)$, but since $F \subset C$, we have $f(x) \vee g(x)>h(x)$. As a result, $f(x)>h(x)$. To show $\phi_{g}\left(\left(K_{1}^{c}\right)^{+}\right) \subset\left(K^{c}\right)^{+}$, fix $f^{*} \in\left(K_{1}^{c}\right)^{+}$. As remarked above, we need to show $f^{*} \vee g$ exceeds $h$ on $C$. Now $f^{*}$ exceeds $h$ on $F$; so, if $x \in F$, then

$$
\left(f^{*} \vee g\right)(x) \geq f^{*}(x)>h(x) .
$$

On the other hand, if $x \in C-F$, then $g(x)>h(x)$ and

$$
\left(f^{*} \vee g\right)(x) \geq g(x)>h(x) \text {. }
$$

It remains to show that for each open subset $W$ of $X \times R, \phi_{g}^{-1}\left(W^{-}\right)$is open in $L(X)$. Suppose $f \vee g \in W^{-}$, i.e., epi $f \cap \operatorname{epi} g \cap W \neq \varnothing$. Since $W$ is open and $g$ is continuous, there exists an open subset $W_{1}$ of $X \times R$ with $W_{1} \subset$ epi $g \cap W$ and epi $f \cap W_{1} \neq \varnothing$. It easily follows that $f \in W_{1}^{-}$and $\phi_{g}\left(W_{1}^{-}\right) \subset W^{-}$. 
LEMMA C. Let $X$ be a completely regular Hausdorff space with at least two points, and let $\tau$ be a topology on $L(X)$ with the properties:

(1) $\arg \min :\langle L(X), \tau\rangle \rightarrow X$ has closed graph;

(2) for each $g \in C(X)$, both $f \rightarrow f \wedge g$ and $f \rightarrow f \vee g$ are continuous operators on $\langle L(X), \tau\rangle$.

Then $\tau$ contains the epi-topology $\tau_{e}$.

Proof. Suppose for some open set $W$ in $X \times R, \tau$ fails to contain $W^{-}$. Then there exists a net $\left\langle f_{\lambda}\right\rangle$ in $L(X) \tau$-convergent to some $f$ in $L(X)$ such that for each index $\lambda, f_{\lambda} \notin W^{-}$, but $f \in W^{-}$. We may assume without loss of generality that $W=U \times(\alpha, \beta)$ where $U$ is a nonempty proper open subset of $X$. The condition $f_{\lambda} \notin W^{-}$says that $f_{\lambda}(u) \geq \beta$ for each $u \in U$. Since epi $f \cap W \neq \varnothing$, there exists $x_{0} \in U$ with $f\left(x_{0}\right)<\beta$. By complete regularity, there exists a continuous function $g: X \rightarrow[\alpha, \beta]$ with $g\left(x_{0}\right)=\alpha$ and $g\left(U^{c}\right)=\beta$. Letting $\beta$ denote the function on $X$ constantly equal to $\beta$, write $f_{\lambda}^{*}=\beta \wedge\left(f_{\lambda} \vee g\right)$ and $f^{*}=\beta \wedge(f \vee g)$. By $(2),\left\langle f_{\lambda}^{*}\right\rangle$ is $\tau$-convergent to $f^{*}$. Let $z \in U^{c}$ be arbitrary. Then $\left(f^{*}, z\right)$ is not in the graph of arg min because

whereas

$$
f^{*}(z)=\beta \wedge(f(z) \vee g(z)) \geq \beta \wedge g(z)=\beta,
$$

$$
f^{*}\left(x_{0}\right)=\beta \wedge\left(f\left(x_{0}\right) \vee g\left(x_{0}\right)\right)=\beta \wedge\left(f\left(x_{0}\right) \vee \alpha\right)<\beta
$$

On the other hand, $f_{\lambda}^{*}$ is just the constant function $\beta$ for each index $\lambda$. As a result, $\arg \min f_{\lambda}^{*}=X$ so that $\left(f_{\lambda}^{*}, z\right)$ lies in the graph of $\arg \min$. We have shown that (1) fails, a contradiction. Thus, for each open subset $W$ of $X \times R$, we must have $W^{-} \in \tau$.

To show that $\tau$ contains the other kind of $\tau_{e}$-subbasic open set is only a little harder. Suppose $K$ is a compact subset of $X \times R$ such that $\left(K^{c}\right)^{+}$is not $\tau$-open. Then we can find a net $\left\langle f_{\lambda}\right\rangle$ in $L(X) \tau$-convergent to some $f \in L(X)$ such that for each $\lambda$, epi $f_{\lambda} \cap K \neq \varnothing$, but epi $f \cap K=\varnothing$. Since $K$ is compact, we can find $\left(x_{0}, \theta\right) \in K$ each neighborhood of which meets the net $\left\langle\right.$ epi $\left.f_{\lambda} \cap K\right\rangle$ frequently. Let $W$ be an arbitrary neighborhood of $\left(x_{0}, \theta\right)$. Since epi $f$ is closed and $X$ is regular, there exists a closed neighborhood $F$ of $x_{0}$ with $F \neq X$ and real numbers $\alpha$ and $\beta$ with $\alpha<\theta<\beta$ such that $F \times[\alpha, \beta] \subset W$ and $(F \times[\alpha, \beta]) \cap$ epi $f=\varnothing$. Let $C=F \cap \pi_{X}(K)$. Since $C$ is compact and $f$ is lower semicontinuous, there exists $\gamma>\beta$ such that $\inf \{f(x): x \in C\}>\gamma$. Let $w \in C^{c}$ be arbitrary; by complete regularity, there exists a continuous function $h: X \rightarrow[\gamma, \gamma+1]$ such that $h(w)=\gamma$ and $h(C)=\gamma+1$. Proceeding as in the first part of the proof, for each index $\lambda$, let $f_{\lambda}^{*}=\left(f_{\lambda} \vee \beta\right) \wedge h$ and let $f^{*}=(f \vee \beta) \wedge h$. By condition $(2),\left\langle f_{\lambda}^{*}\right\rangle \tau$-converges to $f^{*}$. Now for each $\lambda$ we have

$$
\inf \left\{f_{\lambda}^{*}(x): x \in X\right\} \geq \inf \{\beta \wedge h(x): x \in X\}=\beta .
$$

Hence, if $f_{\lambda}^{*}(x)=\beta$, then $x$ will be a minimizer of $f_{\lambda}^{*}$.

We claim that (i) $\left(f^{*}, x_{0}\right)$ lies in the closure of the graph of the arg min multifunction, but (ii) $x_{0} \notin \arg \min f^{*}$. To prove (i), it suffices to show that the closed neighborhood $F$ of $x_{0}$ mentioned above meets $\arg \min f_{\lambda}^{*}$ frequently. To this end, fix an index $\lambda_{0}$ in the underlying directed set for the net. The point $\left(x_{0}, \theta\right)$ was chosen so that each neighborhood of it meets $\left\langle\right.$ epi $\left.f_{\lambda} \cap K\right\rangle$ frequently; so, there exists $\lambda>\lambda_{0}$ for which

$$
(F \times[\alpha, \beta]) \cap\left(\text { epi } f_{\lambda} \cap K\right) \neq \varnothing .
$$


Let $z_{\lambda}$ be in the $X$-projection of this set; then $z_{\lambda} \in C=\pi_{X}(K) \cap F$ and $f_{\lambda}\left(z_{\lambda}\right) \leq \beta$. As a result,

$$
f_{\lambda}^{*}\left(z_{\lambda}\right)=\left(f_{\lambda}\left(z_{\lambda}\right) \vee \beta\right) \wedge h\left(z_{\lambda}\right)=\beta \wedge(\gamma+1)=\beta .
$$

Thus, $z_{\lambda} \in \arg \min f_{\lambda}^{*}$, establishing (i).

For (ii), we must show that $f^{*}\left(x_{0}\right)$ is not the minimal value of $f^{*}$. Since $x_{0}$ lies in $C$, we have $f\left(x_{0}\right)>\gamma$ and $h\left(x_{0}\right)=\gamma+1$; so,

$$
f^{*}\left(x_{0}\right)=\left(f\left(x_{0}\right) \vee \beta\right) \wedge h\left(x_{0}\right)>(\gamma \vee \beta) \wedge(\gamma+1)=\gamma .
$$

On the other hand, $f^{*}(w)=(f(w) \vee \beta) \wedge \gamma \leq \gamma$; so, $f^{*}\left(x_{0}\right)$ is not the minimal value of $f^{*}$.

Putting together Theorem 1 and the lemmas, we have our main result.

THEOREM 2. Let $L(X)$ be the lower semicontinous functions on a locally compact Hausdorff space $X$ with at least two points. Then the epi-topology on $L(X)$ is the weakest topology on the function space such that $\arg \min : L(X) \rightarrow X$ has closed graph, and the operators $f \rightarrow f \wedge g$ and $f \rightarrow f \vee g$ are continuous for each continuous real valued function $g$.

We mention in closing that there is another characterization of the epi-topology, due to Vervaat [14], whose interest in the topology arises from the role it plays in the theory of random semicontinuous functions [15]. Vervaat has shown that it is the weakest topology on $L(X)$ for which a certain class of extended real valued functions on $L(X)$ are lower semicontinuous. Specifically, it is the weakest topology such that for each compact subset $K$ of $X$ and each open subset $U$ of $X$, both

$$
f \rightarrow \inf \{f(x): x \in K\} \quad \text { and } \quad f \rightarrow-\inf \{f(x): x \in U\}
$$

are in $L(L(X))$.

ACKNOWLEDGMENT. The first author's research was supported under the auspices of the National Academy of Sciences Interacademy Exchange Program (April 1986). He is grateful to the second author, and to the Bulgarian Academy of Sciences, for their hospitality.

\section{REFERENCES}

1. H. Attouch, Variational convergence for functions and operators, Pitman, London, 1984.

2. H. Attouch and R. Wets, Approximation and convergence in nonlinear optimization, Nonlinear Programming 4 (O. Mangarasian, Ed.), Academic Press, New York, 1981, pp. 367-394.

3. D. Dal Maso, Questioni di topologie legate allo $\tau$-convergenza, Ricerche Mat. 32 (1983), 135 162.

4. E. De Giorgi, Sulla convergenza di alcune successioni di integrali del tipo del l'area, Rend. Mat. Univ. Roma 8 (1975), 277294.

5. S. Dolecki, G. Salinetti and R. Wets, Convergence of functions: equi-semicontinuity, Trans. Amer. Math. Soc. 276 (1983), 409-429.

6. J. Dugundji, Topology, Allyn and Bacon, Boston, Mass., 1966.

7. E. Effros, Convergence of closed subsets in a topological space, Proc. Amer. Math. Soc. 16 (1965), 929-931.

8. J. M. G. Fell, A Hausdorff topology for the closed subsets of a locally compact non-Hausdorff space, Proc. Amer. Math. Soc. 13 (1962), 472476.

9. J. L. Kelley, General topology, Van Nostrand, Princeton, N. J., 1955.

10. E. Klein and A. Thompson, Theory of correspondences, Wiley, Toronto, 1984. 
11. R. T. Rockafellar and R. Wets, Variational systems, an introduction, Multifunctions and Integrands (G. Salinetti, Ed.), Lecture Notes in Math., vol. 1091, Springer-Verlag, Berlin, 1984.

12. H. Royden, Real analysis, Macmillan, New York, 1968.

13. B. Van Cutsem, Problems of convergence in stochastic linear programming, Techniques of Optimization (A. Balakrishnan, Ed.), Academic Press, New York, 1972, pp. 445-454.

14. W. Vervaat, Une compactification des espaces fonctionnels $C$ et $D$; une alternative pour la démonstration des théorèmes limites fonctionnels, C. R. Acad. Sci. Paris 292 (1981), 441-444.

15. __ Stationary self-similar extremal processes and random semicontinuous functions, Dependence in Probability and Statistics (E. Eberlein and M. Taggu, Eds.), Birkhäuser, Boston, Mass., 1986.

16. R. Wets, Convergence of convex functions, variational inequalities, and convex optimization problems, Variational Inequalities and Complementarity Problems (R. Cottle, F. Gianessi, and J.-L. Lions, Eds.), Wiley, Chichester, U.K., 1980, pp. 375-403.

17. R. Wijsman, Convergence of sequences of convex sets, cones, and functions. II, Trans. Amer. Math. Soc. 123 (1966), 32-45.

18. T. Zolezzi, Characterization of some variational perturbations of the abstract linear-quadratic problem, SIAM J. Control Optim. 16 (1978), 106-121.

Department of Mathematics, California State University, los Angeles, CALIFORNIA 90032

Institute of Mathematics, Bulgarian ACAdemy of Sciences, 1113, Sofia, BulGARIA 